\title{
Clinical Course of Multiple Sclerosis
}

\author{
Sylvia Klineova and Fred D. Lublin \\ The CGD Center for Multiple Sclerosis, Icahn School of Medicine at Mount Sinai, New York, New York 10029 \\ Correspondence: sylvia.klineova@mssm.edu
}

The 1996 originally established multiple sclerosis (MS) subtypes, based solely on clinical impression and consensus, were revised in 2013 to review potential imaging and biological correlates and to reflect recently identified clinical aspects of MS. As a result, potential new disease phenotypes, radiologically isolated syndrome, and clinically isolated syndrome were considered along with the addition of two new descriptor subtypes: activity and progression applied to relapsing remitting and progressive MS phenotypes. In this way, the description of an individual patient's disease course is refined and provides temporal information about the ongoing disease process. There is still a lack of imaging and biological markers that would distinguish MS phenotypes and prognosticate the disease course on an individual patient's level, creating a pressing need for large collaborative research efforts in this field.

$M$ ultiple sclerosis (MS), a chronic, inflammatory disease of the central nervous system (CNS) with hallmarks of demyelination and axonal degeneration, is characterized by heterogeneity in the symptoms, disease course, and outcomes (Compston and Coles 2008). Typically affecting patients between 20 and 40 years of age, MS is a leading cause of disability in young adults in the United States and Europe (Tullman 2013).

MS is considered to be an autoimmune disease mediated by autoreactive $\mathrm{T}$ helper (Th) 1 and Th17 cells. Initial contact with a yet-unknown antigen leads to production of proinflammatory cytokines, interleukin (IL)-1, and interferon (IFN) $-\gamma$ by Th1 cells and IL-17 by Th17 cells. Production of cytokines leads to further Th cell up-regulation, production of certain metalloproteinases, and destruction of the blood-brain barrier (BBB), allowing Th cells to migrate into the CNS. Recovery may be mediated by distinctive Th 2 cell populations secreting IL-10 and -4 anti-inflammatory cytokines (Dhib-Jalbut 2002; Sie et al. 2014). Although T cells have long been considered integral to MS pathogenesis, B-cell follicles and oligoclonal bands (OCBs) are also present in the MS CNS, and it is becoming increasingly clear that $\mathrm{B}$ cells contribute to MS through mechanisms more complex than previously appreciated (Cross et al. 2001).

The first formally defined MS phenotypes, relapsing remitting MS (RRMS), primary progressive MS (PPMS), secondary progressive MS (SPMS), and progressive relapsing MS (PRMS), were proposed in 1996 by the U.S. National Multiple Sclerosis Society (NMSS) Advisory Committee on Clinical Trials in Multiple Sclerosis as a result of increased need for standardized terminology in the field. It was felt that lack

Editors: Howard L. Weiner and Vijay K. Kuchroo

Additional Perspectives on Multiple Sclerosis available at www.perspectivesinmedicine.org

Copyright (C) 2018 Cold Spring Harbor Laboratory Press; all rights reserved; doi: 10.1101/cshperspect.a028928

Cite this article as Cold Spring Harb Perspect Med 2018;8:a028928 
of such terminology would be detrimental not only to clinical practice and communication among clinicians but also to future advances in clinical research. The unified terminology would ensure the proper design of clinical trials, ensure the homogeneity of population recruited, and thus provide the necessary groundwork for successful outcomes. However, the Committee also recognized the purely clinical nature of the phenotypes and acknowledged that this terminology might change over time (Lublin and Reingold 1996).

The proposed nomenclature quickly became an inherent part of clinical and research practice and was partly instrumental in the approval process of new MS therapeutics. However, with the increased knowledge base of MS pathology, the limitations of purely clinical phenotypes, lacking imaging and biological correlates, became evident. In 2012, the Committee (sponsored by NMSS and the European Committee for Treatment and Research in MS) reexamined the original clinical phenotypes with a goal to provide improved terminology while incorporating imaging, fluid biomarkers, and other assays. The Committee recommended retention of the basics of the original 1996 MS phenotypes but provided enhanced characterization by introducing new descriptors of activity and progression. The Committee also reported on two new disease courses: radiologically isolated syndrome (RIS) and clinically isolated syndrome (CIS) (Table 1) (Lublin et al. 2014).

We will review currently recognized and new MS disease courses.

\section{RADIOLOGICALLY ISOLATED SYNDROME}

Although RIS is not considered a distinct MS phenotype (Lublin et al. 2014), increasing frequency of this incidental magnetic resonance imaging (MRI) finding in patients has raised its awareness in the MS community.

The term RIS, first introduced in 2009 (Okuda et al. 2009), identifies patients with incidentally found MRI abnormalities highly suggestive of demyelination in the absence of clinical signs or symptoms. The proposed diagnostic criteria used the original Barkhof criteria
(Barkhof et al. 1997) for dissemination in spaceand lesion-specific morphologic features to enhance diagnostic certainty and eliminate patients with nonspecific white matter changes caused by other causative etiologies, such as vascular disease or migraines. As those patients are at increased risk of developing clinically definitive MS (CDMS) in the future, identifying baseline factors with prognostic relevance for future long-term outcomes is of high importance.

Few studies explored the natural disease course in RIS patients and attempted to identify risk factors for either clinical or radiological disease progression.

In 2009, Okuda reported outcomes in 44 RIS patients with regard to conversion to CIS or radiological progression. The study showed a $30 \%$ conversion rate to CIS or CDMS with a median time of 5.4 years to the event. Radiological progression was found in $59 \%$ of patients over a median time of 2.7 years. The presence of gadolinium-enhancing lesions on the baseline MRI substantially increased the risk for radiological progression (hazard ratio $[\mathrm{HR}]=3.4$ ) (Okuda et al. 2009).

Another prospective study by French investigators also from 2009 studied outcomes in 70 RIS patients.

In agreement with the previous study, the investigators found a $37 \%$ CIS conversion rate among the subjects over a mean follow-up period of 5.2 years. The mean time to a second MRI was 6 months and radiological progression was found in $91 \%$ of patients, with $37 \%$ of patients developing gad-enhancing lesions. With regard to other baseline predictive factors, this study found a significant impact of positive OCBs or an increased immunoglobulin G (IgG) index but only when associated with $\geq 9$ T2-hyperintense lesions on a first MRI (Lebrun et al. 2009).

The largest study reporting on RIS disease course to date was a retrospective, multinational cohort study that in 2014 reported on 451 RIS subjects with the goal of estimating the 5-year risk of developing a first clinical event while also investigating the predictive validity of any associated demographic, clinical, and radiological risk factors. Similar to the two previous studies, the investigators found a $34 \%$ risk of first acute 
Clinical Course of MS

Table 1. Changes in multiple sclerosis disease-course (or "type") descriptions

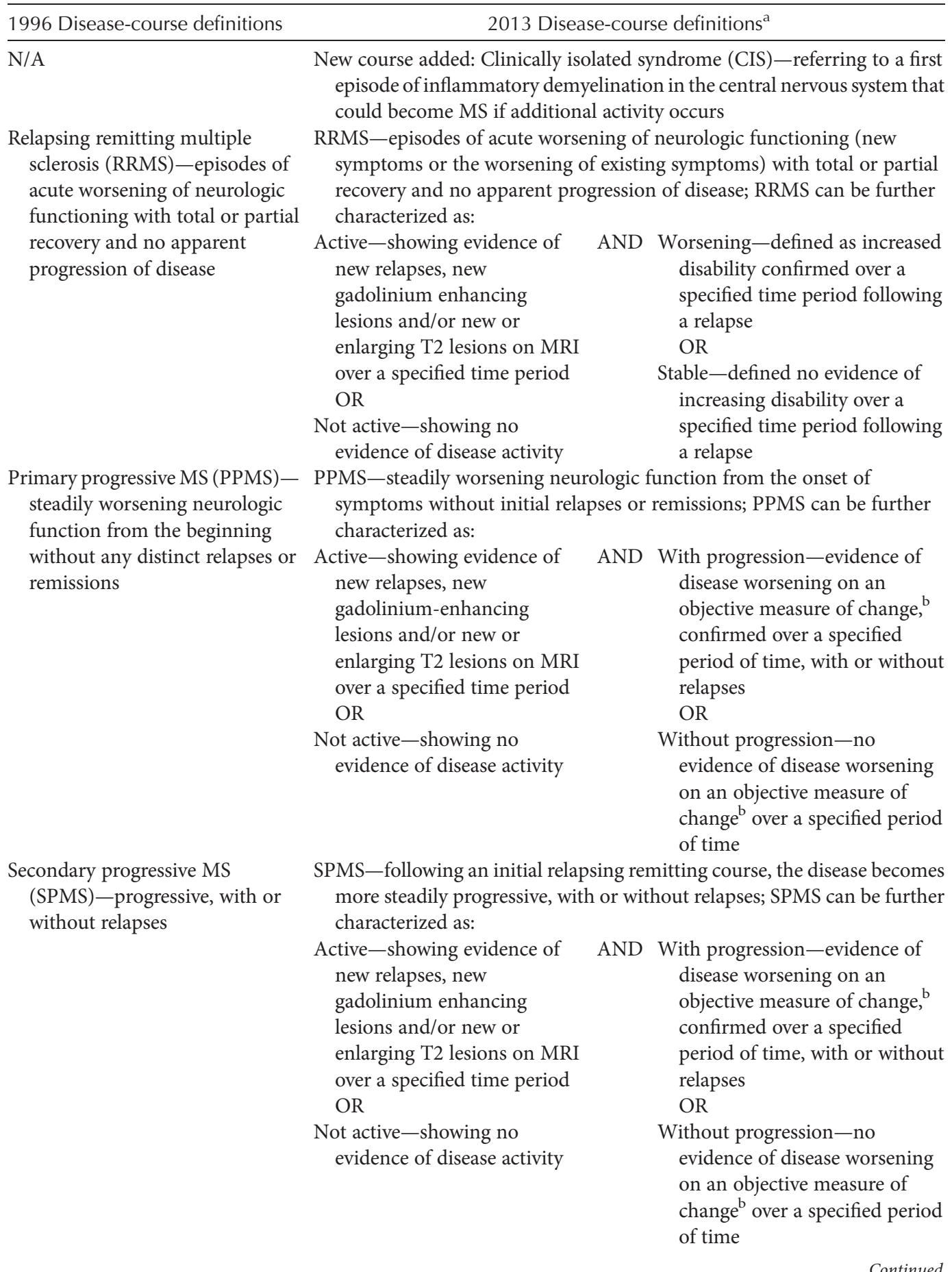

Continued 
Table 1. Continued

\begin{tabular}{ll}
\hline 1996 Disease-course definitions & 2013 Disease-course definitions ${ }^{\text {a }}$ \\
\hline $\begin{array}{ll}\text { Progressive relapsing MS (PRMS) - } \\
\text { steadily worsening neurologic } \\
\begin{array}{l}\text { function from the beginning with } \\
\text { occasional relapses }\end{array}\end{array}$ & $\begin{array}{l}\text { with progressive relapsing MS would now be considered primary } \\
\text { progressive: active (at the time of relapses or new MRI lesions) or not } \\
\text { active }\end{array}$ \\
\hline
\end{tabular}

Based on data from the National Multiple Sclerosis Society. MRI, Magnetic resonance imaging.

${ }^{a}$ Lublin et al. (2014).

${ }^{\mathrm{b}}$ For example, the Expanded Disability Status Scale (EDSS).

or progressive clinical event observed within 5 years. A unique observation in this study was that $9.6 \%$ of patients developed a progressive disease course from onset. This study also defined spinal cord lesions as the strongest predictive factor for future clinical events $(\mathrm{HR}=3.08)$. Additional relevant factors were younger age and male gender. Risk also increased with the presence of multiple risk factors. Interestingly, factors known for their importance in CIS, such as gad-enhancing lesions, infratentorial lesions, and positive cerebrospinal fluid (CSF) profile were not predictive of first clinical event development. Bearing in mind that this study was not investigating the impact of treatment on the risk of first clinical event (16.2\% of patients in the cohort were treated with disease-modifying treatments [DMTs]), the investigators found no benefit of DMT agents on extending the time period to the first event (Okuda et al. 2014).

The importance of RIS and the need for accurate characterization of its course and associated risks is supported not only by the fact that a meaningful number of patients do convert to clinical MS but also by the concern for misdiagnosis and eventual exposure to treatment agents. Although still not yet recognized as a formal MS course, research efforts to validate the abovereported findings and to establish the role of DMT treatment in RIS are under way.

\section{CLINICALLY ISOLATED SYNDROME}

Although recognized for some time, the 2012 MS disease course nomenclature codified CIS as an established disease course.

The term CIS describes a first clinical event highly suggestive of demyelinating CNS disease but not yet meeting dissemination in time for diagnosis of CDMS. The presenting symptoms are usually monofocal, evolve acutely or subacutely over days to weeks, and involve optic nerve, spinal cord, brain stem, or cerebellum. Like other MS attacks, the episode is expected to last at least 24 hours and occurs in the absence of fever or infection (Miller et al. 2012).

The CIS harbors a possibility of CDMS in the future. The relationship between CIS and MS was the subject of few observational studies that reported on a conversion rate to CDMS following different CIS events: optic neuritis, brain stem syndromes, and transverse myelitis. Despite the variations in the conversion rates observed (CDMS conversion after optic neuritis up to $85 \%$, transverse myelitis up to $61 \%$, and brain stem syndromes up to $60 \%$ ), most likely related to geographical differences in the course of the disease and different follow-up periods, it is safe to conclude that CDMS risk rates are similar for all the CIS types (Optic Neuritis Study Group 2008; Young et al. 2009; Tintore et al. 2010).

Similar to RIS, certain demographic and imaging characteristics influence the risk of clinically definite multiple sclerosis (CDMD) conversion. The presence and number of T2 white matter lesions and abnormal CSF profile, defined as an elevated IgG index or the presence of OCBs, are the two predictors most commonly used in clinical practice.

The majority of CIS patients (50\%-70\%) will have asymptomatic $\mathrm{T} 2$ white matter abnormalities on the baseline brain MRI consistent with demyelinating lesions. The predictive role of this finding with regard to CDMS conversion was reported in multiple observational long- 
term studies and showed up to $80 \%$ conversion rate in up to 20 years follow-up period. The risk of CDMS conversion is also correlated with the number of lesions (Brex et al. 2002; Tintore et al. 2010; Miller et al. 2012).

Although the presence of abnormal CSF profile did not provide much added prognostic value to the presence of abnormal MRI in CIS patients, the predictive value further increased in patients with normal brain MRI, raising the risk to CDMS conversion from $4 \%$ to $23 \%$ (Tintore et al. 2008).

\section{RELAPSING REMITTING MS}

The most common MS phenotype, found in about $85 \%$ of MS patients, RRMS, is characterized by alternating periods of neurological dysfunction-relapses and periods of relative clinical stability free of new neurological symptoms -remissions (Fig. 1). The frequency of relapses can vary from patient to patient but generally does not exceed 1.5 per year. Various neurological symptoms, such as weakness, altered sensation, balance impairment, impairment of visual acuity, and color vision or double vision, can be present during the relapse, lasting at least 24 hours in the absence of infection or metabolic derangement. Relapses result in residual deficits in almost half of episodes, leading to stepwise accrual of impairment (Lublin et al. 2003).

Pathologically, areas of inflammation rich in perivascular lymphocytic infiltrates with subsequent demyelination and axonal transection are the substrate of a relapse episode. Recovery of the symptoms during the relapse resolution suggests remyelination, which is most active during the early inflammatory phase of MS (Compston and Coles 2008).

The magnitude of inflammatory pathology and frequency of relapses, most prominent in young adulthood, decreases with advanced disease and age. One of the natural disease history studies found a relapse rate of 0.54 associated with average disease duration of 16 years along with decreased duration of second remission from 71.32 months to 58.07 months (Boiko et al. 2002).

Many potential relapse triggers have been investigated over the years to identify potential interventions to prevent an acute attack. Infections and stress, as well as pregnancy and their association with relapse, are the most relevant factors to everyday clinical practice.

Studies show an association between infections and increased relapse rate, prolonged relapse duration, and increased accumulation of disability; but no specific pathogen has yet

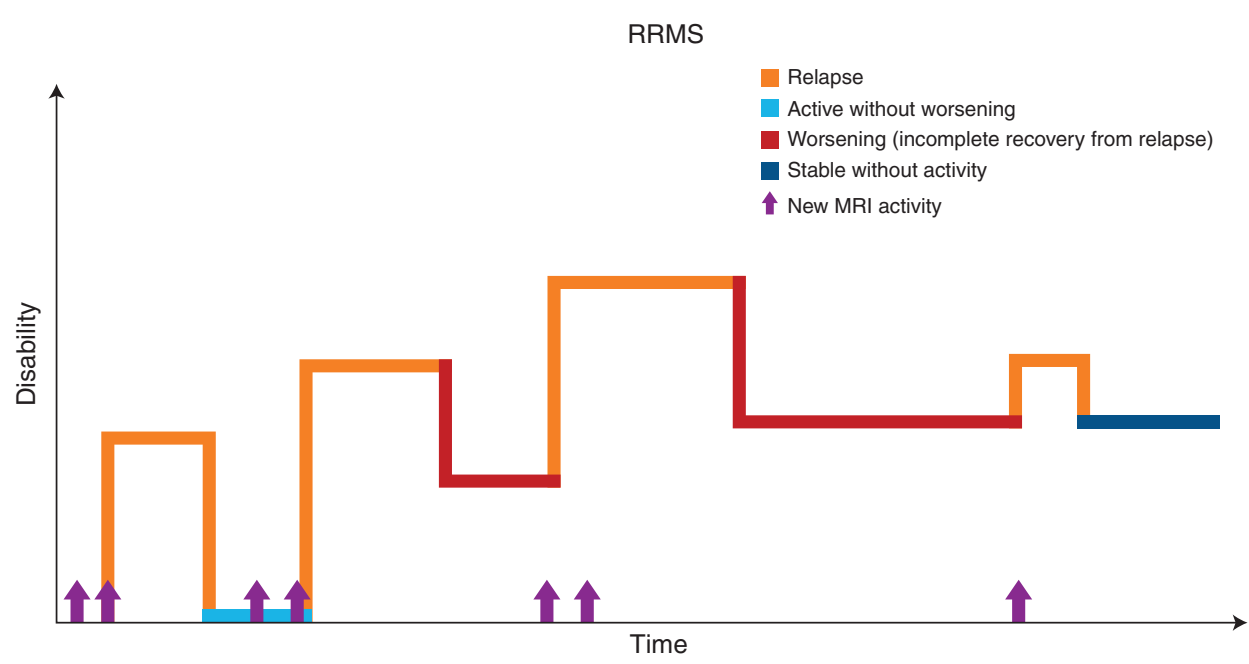

Figure 1. Disease course of relapsing remitting multiple sclerosis (RRMS). (Reprinted, with permission, from the National Multiple Sclerosis Society.) 
been identified. Upper respiratory infections have been most often reported as potential triggers, but urinary tract infections and gastroenteritis are also associated with an increased relative risk for relapse (Vollmer 2007). Although many patients report stressful events as a trigger for MS relapse, the review of literature by Mohr et al. (2004) did not find a strong causative relationship and proposed a rather additive role of stress to many other factors influencing relapse onset.

Pregnancy and associated biochemical changes are known to affect the relapse rate in MS. A study by the Pregnancy in Multiple Sclerosis Group showed a decreasing relapse rate during pregnancy, from 0.7 in the year before pregnancy to 0.5 , and 0.6 and 0.2 during the subsequent trimesters. The relapse rate increased in the immediate postpartum period of 3 months to 1.2 before returning to 0.6 by the end of the postpartum year. This effect is believed to be caused by the natural immunotolerant effect of pregnancy, which is then lost at the time of delivery (Confavreux et al. 1998).

Knowing that a significant number of the RRMS patients will eventually progress into SPMS; can relapse characteristics serve as a surrogate marker to predict the time of onset and degree of future progression? The research in this field showed conflicting results. Although several studies showed no effect of relapses, either before the progression or superimposed, on the severity of the progression, others clearly showed the opposite.

Using a natural history cohort, a study by Weinshenker et al. (1989) showed that the number of relapses within first 2 years of diagnosis did influence the median time of onset of the progressive disease. Another study, a systematic review of predictors for long-term disability identified the short interrelapse period after the first attack to be the strongest predictor for the time to onset of disability (LangerGould et al. 2006). Work by Paz Soldan et al. (2015) also showed that pre- and postprogressive relapses independently accelerated the time to severe disability in progressive MS.

In concordance with these results, the study by French investigators showed slower onset of progression in patients with RRMS when compared with PPMS, but once the threshold of a progression was reached, both groups behaved in a similar fashion (Confavreux et al. 2000).

In 2006, a large Canadian cohort study from London, Ontario, following more than $1000 \mathrm{pa}-$ tients with RRMS, did not confirm the relapse frequency as a substantial surrogate marker for the future progression. The investigators found no effect of the relapse rate on the slope of the progressive disease. The study showed dissociation between relapses and disease progression, evidenced by parallel progression of disability in both PPMS and SPMS with or without relapses. Using the same Canadian cohort in a subsequent study in 2009, the investigators found that frequent early attacks (first 2 years from onset) lead to earlier onset of progressive disease (Kremenchutzky et al. 2006; Scalfari et al. 2013).

Differences in methodology and data interpretation are the possible reasons for such contradicting views on this topic; however, the importance of relapses on future disability should be recognized as it directly influences treatment strategies (Lublin 2011).

\section{SECONDARY PROGRESSIVE MS}

The majority of untreated RRMS patients do eventually progress into SPMS, and research data suggest a median time to progressive phase of about 19 years after the onset of RRMS (Rovaris et al. 2006). The diagnosis is most often established retrospectively, years after the actual progression started. On an individual patient level, it is difficult to determine when exactly in the disease course does the transition start, and patients as well as clinicians can encounter several years of diagnostic uncertainty. The most common reasons for this period of uncertainty, reported to last $2.9 \pm 0.8$ years on average in one examined population (Katz Sand et al. 2014), are subtle and often fluctuating initial symptoms indicating early progression and reluctance to establish a diagnosis of progressive disease and thus increasing patient's anxiety regarding prognosis and lack of approved therapies.

A few predictors of the conversion to SPMS have been identified, namely, higher age at 
RRMS onset was associated with earlier progression to SPMS, as well as male gender, albeit not consistently in all studies. Spinal cord symptoms and incomplete relapse recovery have also been shown to shorten the time to progression (Rovaris et al. 2006).

Phenotypically, the course of SPMS is not uniform and consists of periods of progression with possible superimposed relapse activity but also periods of relatively stable disability (Fig. 2). To date, there are no available imaging or immunological markers of progression, which is estimated based on clinical grounds over a period of at least 6 to 12 months.

The pathology involved in SPMS is poorly understood and most likely complex, involving some degree of persistent inflammation, albeit to a lesser extent than in RRMS, combined with neurodegeneration caused by mitochondrial dysfunction and resultant axonal damage.

The peripheral innate immune system composed of the cells of myeloid origin, such as dendritic cells, macrophages, and natural killer cells, is now recognized to play an important role in mediating and regulating pathology in MS, notably in the progressive phase. A shift from adaptive to innate immunity has been proposed as a potential mechanism of progression. Recent research has shown changes in the cytokines (IL-12 and -18) and costimulatory molecules in the dendritic cells of patients transitioning from RRMS to SPMS. Additional processes leading to neuronal and oligodendrocyte cell death may include nitric oxide production and respiratory burst as well as secretion of soluble proteins (matrix metalloproteinases) with a direct effect on the $\mathrm{BBB}$ (Weiner 2008).

The inflammatory changes present in progressive disease are thought to be compartmentalized within the CNS behind a closed or repaired $\mathrm{BBB}$. This compartmentalized inflammation is the postulated driving force behind expansion of existing lesions and diffuse changes in normally appearing white matter. In addition, focal areas of inflammation can be also found within the meninges of progressive patients in lymphoid follicle-like structures containing dense clusters of B cells and plasma cells, possibly causing the higher degree of cortical pathology observed in progressive disease (Lassmann et al. 2007).

The reorganization of voltage-gated sodium channels along demyelinated axons leading to increased energy requirements, failing ATP production, and accumulation of intracellular calcium resulting in mitochondrial dysfunction and axonal damage is a proposed mechanism explaining the role of mitochondria in neurodegeneration (Su et al. 2009).

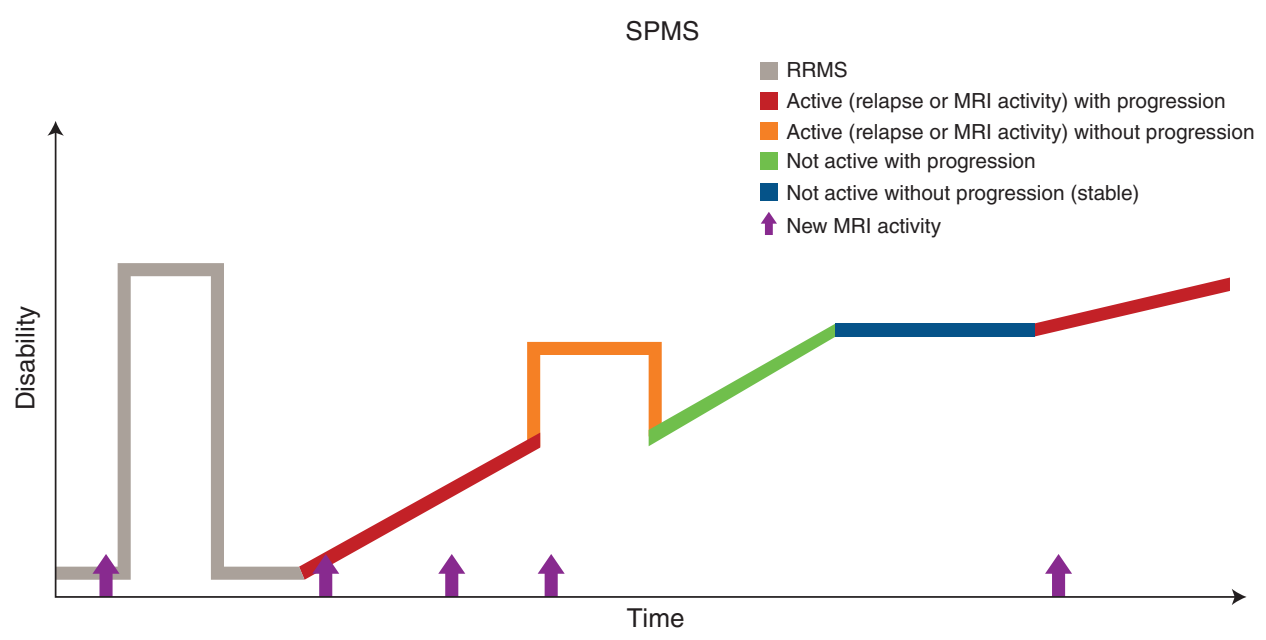

Figure 2. Disease course of secondary progressive multiple sclerosis (SPMS). RRMS, Relapsing remitting multiple sclerosis; MRI, magnetic resonance imaging. (Reprinted, with permission, from the National Multiple Sclerosis Society.) 
S. Klineova and F.D. Lublin

PPMS

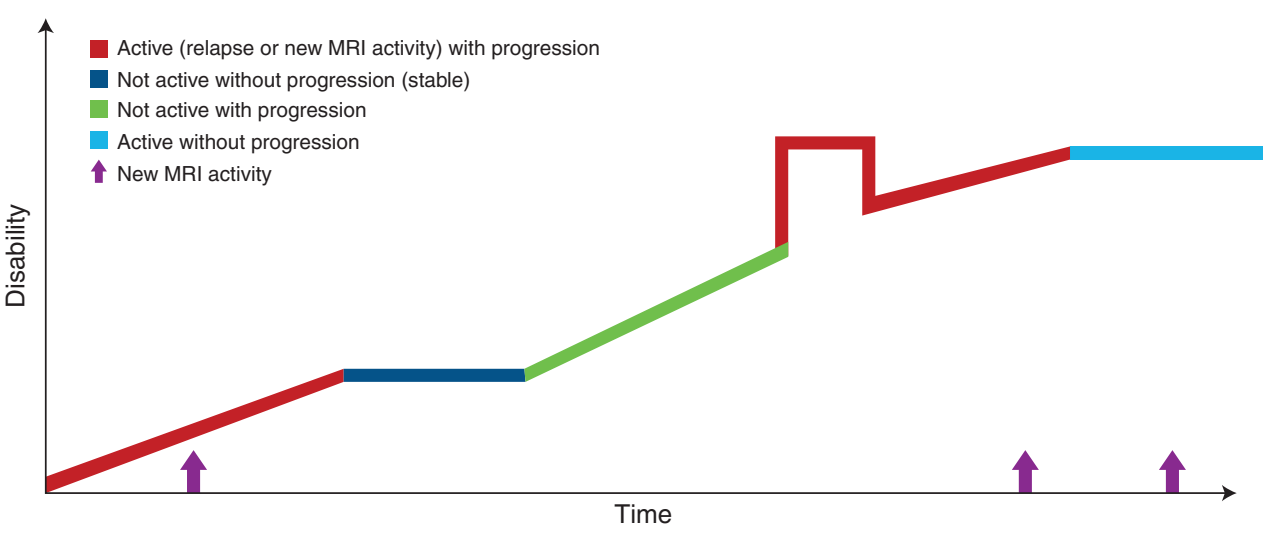

Figure 3. Disease course of primary progressive multiple sclerosis (PPMS). MRI, Magnetic resonance imaging. (Reprinted, with permission, from the National Multiple Sclerosis Society.)

\section{PRIMARY PROGRESSIVE MS}

About $10 \%-20 \%$ of patients will develop this disease phenotype, characterized by the lack of initial RR phase and ongoing progression from the disease onset (Compston and Coles 2008; Ransohoff et al. 2015). On an individual patient level, progression is not uniform throughout the course and superimposed relapses as well as periods of relative disease stability are possible (Fig. 3).

Increasing clinical, imaging, and genetic data suggest that PPMS is a part of the MS disease spectrum and any pathological differences from SPMS are relative rather than absolute. Natural history studies have shown that disability progresses in parallel in patients with PPMS and SPMS with or without relapses (Kremenchutzky et al. 2006). The fact that $\sim 10 \%$ of RIS patients develop a PP disease course further supports the theory that the absence of RR phase in PPMS patients is potentially caused by clinically silent CNS lesions (Okuda et al. 2014; Ransohoff et al. 2015).

Similar to SPMS, pathology in PPMS is complex and includes neurodegeneration occurring along with mild-to-moderate inflammation.

\section{MODIFIERS OF ACTIVITY AND PROGRESSION IN MS}

The addition of two new modifiers, activity and progression, to the established MS phenotypes, was proposed in the 2013 revision based on an increased understanding of the MS clinical course and the increasingly important role of MRI in clinical care and research. While the 1996 MS phenotypes provide a more static disease description, addition of the descriptors of activity and progression enhances characterization of the ongoing disease dynamic in a given time period and enhances prognostication, treatment decisions, and outcomes in clinical care as well as research.

Disease activity is defined as a clinical relapse or new MRI activity-presence of gad-enhancing lesions or new/enlarging T2 lesions. The descriptor of disease activity applies to relapsing and progressive MS patients. The Committee suggested yearly assessment of disease activity using the clinical examination in both RRMS and progressive MS patients. Annual assessment of MRI activity in relapsing patients was deemed satisfactory but no consensus on appropriate MRI frequency in progressive patients was reached. Because of the correlation between brain and spine MRI activity, annual spinal MRI surveillance scans to detect activity were not recommended unless there were new spinal symptoms present (Bot and Barkhof 2009).

Inclusion of the new descriptive terminology then results in various MS phenotypes, such as RRMS-active, in RRMS patients with either clinical or MRI disease activity or PPMS-not 
active in PPMS patients with no acute attacks or MRI activity within a stated time period.

The descriptor of disease progression applies to either SPMS or PPMS patients. As progression in MS is not a uniform feature and patients can remain relatively stable over time, yearly assessment of progression is recommended. Owing to the lack of imaging or immunological biomarkers, the progression of the disease is determined on a clinical basis only, combining objective findings and patient-provided history. Again, combining features of activity and progression, various MS phenotypes can be observed, such as SPMS-active and progressing in SPMS patient with clinical or imaging activity and progression of the disability (Table 1) (Lublin et al. 2014).

The addition of the MS phenotypical modifiers of activity and progression is a first step toward more patient-specific terminology and subsequently toward more individualized care.

The original 1996 MS phenotypes were based on purely clinical grounds and clinical consensus and it was hoped that in the future, biomarkers, either imaging or biological, would be able to better support and define the phenotypes. Yet, to date, none of the many proposed candidates have made it into clinical practice. A review of biomarkers is beyond the scope of this article, but we will briefly review some of them with regard to the MS disease course.

\section{MRI}

The 2010 revision of McDonald diagnostic criteria enhanced the usage of MRIs in the diagnostic process and allowed for earlier RRMS diagnosis and treatment. The importance of an MRI in MS is indisputable but, to date, MRIs are not able to distinguish among MS disease courses.

It is clear that the presence of white matter lesions on MRIs in the correct clinical circumstances defines RIS and CIS disease phenotypes as well as transition from CIS to RRMS. Enhancing or new T2 lesions are now used as one of the markers of activity. However, in established MS, the conventional MRI metrics correlate only moderately with disability measures, caus- ing the radiological and clinical paradox (Filippi and Rocca 2011). This is caused by the relative lack of specificity that conventional MRI metrics have for the heterogeneous pathological substrates of the disease. Novel imaging techniques, such as magnetization transfer ratio (MTR), diffusion inversion recovery (DIR), and diffusion tensor imaging (DTI) have been expected to fill this knowledge gap.

Statistically significant differences in the degree of MTR reduction in T1 hypointense lesions have been reported among patients with RRMS and SPMS (Filippi and Agosta 2007). Additionally, MTR changes found in normally appearing white matter and gray matter evolved in distinguished pattern among major MS phenotypes (Filippi and Agosta 2010). The subtle DTI changes, specifically, increased mean diffusivity, can be found in some patients early before the formation of acute inflammatory lesions.

The introduction of DIR sequences enhanced the ability to detect cortical lesions. Cortical lesions are more frequently seen in SPMS patients than in RRMS or CIS patients, and association has been reported between these lesions and the progression of disability in specific MS phenotypes (Filippi and Rocca 2011).

From the present and ongoing research, as well as our clinical experience, it is safe to say that only combining conventional and unconventional MRI techniques with different spectrums of specificity toward different processes might enhance our understanding of this complex disease and its course. Although this might be a feasible approach in clinical research, it is questionable whether the same is applicable to clinical care.

\section{BIOLOGICAL BIOMARKERS}

There are different requirements for biological biomarkers with regard to identifying an MS subtype (specific) or predicting an MS course (dynamically changing ahead, not after, the transition), but the common expectations are standardized analysis techniques, validation in large independent cohorts, and cost-effectiveness for clinical practice. The ideal biomarker should not be redundant in information already 
provided by MRI imaging, but rather additive, and provide a different spectrum of information.

Many promising biomarkers, either serum or CSF, have been identified but very few were also validated in at least two independent studies, and none of those have made it into clinical practice as yet.

The main areas where disease biomarkers could provide more information are identification of early MS and prediction of CIS to CDMD conversion, identification of MS subtypes and prognostication within and across the MS phenotypes, and response to therapy.

The following biomarkers showed promising results and were validated in at least one independent cohort for identification of early CIS to CDMS converters: CSF IgM OCBs, CSF C-XC motif chemokine 13 (CXCL13), CSF chitinase-3-like protein 1 (CHI3L1), and CSF neurofilament light chain (NfL). Identification of MS subtypes, specifically identification of PPMS versus RRMS, was supported by the presence of serum microRNAs miR-223 and miR-15b. Decreased levels of CSF $N$-acetylaspartate (NAA) were found in patients with SPMS in comparison with RRMS and CIS. With regard to prognostication and intraphenotypical characterization of individual patients, CSF-restricted IgM OCBs were found in patients with high relapse rate and early progression to SPMS, elevated CSF CHI3L1 levels were associated with earlier progression to high Expanded Disability Status Scale (EDSS) scores in RRMS patients (Teunissen et al. 2015).

Development of new biomarkers is a long process and large, collaborative studies are essential for the validation of results before any biomarker makes it into clinical practice. As optimal use of resources is an important factor in any health-care setting, we should consider the usage of information that any biomarker is able to provide and prioritize the most pressing clinical needs-progressive MS and prediction of treatment response.

\section{CONCLUDING REMARKS}

Our increased understanding of MS clinical course changed the view of the originally de- fined MS phenotypes and resulted in new MS subtypes with enhanced characterization of the individual patient disease course. This is the first step into personalized care in MS. New developments in the field of MRI and biomarkers will further pave the way toward a better future for our patients.

\section{REFERENCES}

Barkhof F, Filippi M, Miller DH, Scheltens P, Campi A, Polman CH, Comi G, Ader HJ, Losseff N, Valk J. 1997. Comparison of MRI criteria at first presentation to predict conversion to clinically definite multiple sclerosis. Brain 120: 2059-2069.

Boiko A, Vorobeychik G, Paty D, Devonshire V, Sadovnick D; University of British Columbia MS Clinic Neurologists. 2002. Early onset multiple sclerosis: A longitudinal study. Neurology 59: 1006-1010.

Bot JC, Barkhof F. 2009. Spinal-cord MRI in multiple sclerosis: Conventional and nonconventional MR techniques. Neuroimaging Clin N Am 19: 81-99.

Brex PA, Ciccarelli O, O’Riordan JI, Sailer M, Thompson AJ, Miller DH. 2002. A longitudinal study of abnormalities on MRI and disability from multiple sclerosis. $N \mathrm{Engl} \mathrm{J}$ Med 346: 158-164.

Compston A, Coles A. 2008. Multiple sclerosis. Lancet 372: 1502-1517.

Confavreux C, Hutchinson M, Hours MM, CortinovisTourniaire P, Moreau T. 1998. Rate of pregnancy-related relapse in multiple sclerosis. Pregnancy in Multiple Sclerosis Group. N Engl J Med 339: 285-291.

Confavreux C, Vukusic S, Moreau T, Adeleine P. 2000. Relapses and progression of disability in multiple sclerosis N Engl J Med 343: 1430-1438.

Cross AH, Trotter JL, Lyons J. 2001. B cells and antibodies in CNS demyelinating disease. J Neuroimmunol 112: 1-14.

Dhib-Jalbut S. 2002. Mechanisms of action of interferons and glatiramer acetate in multiple sclerosis. Neurology 58: S3-S9.

Filippi M, Agosta F. 2007. Magnetization transfer MRI in multiple sclerosis. J Neuroimaging 17: 22S-26S.

Filippi M, Agosta F. 2010. Imaging biomarkers in multiple sclerosis. J Magn Reson Imaging 31: 770-788.

Filippi M, Rocca MA. 2011. The role of magnetic resonance imaging in the study of multiple sclerosis: Diagnosis, prognosis and understanding disease pathophysiology. Acta Neurol Belg 111: 89-98.

Katz Sand I, Krieger S, Farrell C, Miller AE. 2014. Diagnostic uncertainty during the transition to secondary progressive multiple sclerosis. Mult Scler 20: 1654-1657.

Kremenchutzky M, Rice GP, Baskerville J, Wingerchuk DM, Ebers GC. 2006. The natural history of multiple sclerosis: A geographically based study 9: Observations on the progressive phase of the disease. Brain 129: 584-594.

Langer-Gould A, Popat RA, Huang SM, Cobb K, Fontoura P, Gould MK, Nelson LM. 2006. Clinical and demographic predictors of long-term disability in patients with relaps- 
ing-remitting multiple sclerosis: A systematic review. Arch Neurol 63: 1686-1691.

Lassmann H, Bruck W, Lucchinetti CF. 2007. The immunopathology of multiple sclerosis: An overview. Brain Pathol 17: $210-218$.

Lebrun C, Bensa C, Debouverie M, Wiertlevski S, Brassat D, de Seze J, Rumbach L, Pelletier J, Labauge P, Brochet B, et al. 2009. Association between clinical conversion to multiple sclerosis in radiologically isolated syndrome and magnetic resonance imaging, cerebrospinal fluid, and visual evoked potential: Follow-up of 70 patients. Arch Neurol 66: 841-846.

Lublin FD. 2011. Relapses do not matter in relation to longterm disability: No (they do). Mult Scler 17: 1415-1416.

Lublin FD, Reingold SC. 1996. Defining the clinical course of multiple sclerosis: Results of an international survey. National Multiple Sclerosis Society (USA) Advisory Committee on Clinical Trials of New Agents in Multiple Sclerosis. Neurology 46: 907-911.

Lublin FD, Baier M, Cutter G. 2003. Effect of relapses on development of residual deficit in multiple sclerosis. $\mathrm{Neu}$ rology 61: 1528-1532.

Lublin FD, Reingold SC, Cohen JA, Cutter GR, Sørensen PS, Thompson AJ, Wolinsky JS, Balcer LJ, Banwell B, Barkhof F, et al. 2014. Defining the clinical course of multiple sclerosis: The 2013 revisions. Neurology 83: 278-286.

Miller DH, Chard DT, Ciccarelli O. 2012. Clinically isolated syndromes. Lancet Neurol 11: 157-169.

Mohr DC, Hart SL, Julian L, Cox D, Pelletier D. 2004. Association between stressful life events and exacerbation in multiple sclerosis: A meta-analysis. BMJ 328: 731.

Okuda DT, Mowry EM, Beheshtian A, Waubant E, Baranzini SE, Goodin DS, Hauser SL, Pelletier D. 2009. Incidental MRI anomalies suggestive of multiple sclerosis: The radiologically isolated syndrome. Neurology 72: 800-805.

Okuda DT, Siva A, Kantarci O, Inglese M, Katz I, Tutuncu M, Keegan BM, Donlon S, Hua LH, Vidal-Jordana A, et al. 2014. Radiologically isolated syndrome: 5 -year risk for an initial clinical event. PLoS ONE 9: e90509.

Optic Neuritis Study Group. 2008. Multiple sclerosis risk after optic neuritis: Final optic neuritis treatment trial follow-up. Arch Neurol 65: 727-732.

Paz Soldan MM, Novotna M, Abou Zeid N, Kale N, Tutuncu M, Crusan DJ, Atkinson EJ, Siva A, Keegan BM, Pirko I, et al. 2015. Relapses and disability accumulation in progressive multiple sclerosis. Neurology 84: 81-88.
Ransohoff RM, Hafler DA, Lucchinetti CF. 2015. Multiple sclerosis-A quiet revolution. Nat Rev Neurol 11: 134142.

Rovaris M, Confavreux C, Furlan R, Kappos L, Comi G, Filippi M. 2006. Secondary progressive multiple sclerosis: Current knowledge and future challenges. Lancet Neurol 5: 343-354.

Scalfari A, Neuhaus A, Daumer M, Deluca GC, Muraro PA, Ebers GC. 2013. Early relapses, onset of progression, and late outcome in multiple sclerosis. JAMA Neurol 70: 214222.

Sie C, Korn T, Mitsdoerffer M. 2014. Th17 cells in central nervous system autoimmunity. Exp Neurol 262: 18-27.

Su KG, Banker G, Bourdette D, Forte M. 2009. Axonal degeneration in multiple sclerosis: The mitochondrial hypothesis. Curr Neurol Neurosci Rep 9: 411-417.

Teunissen CE, Malekzadeh A, Leurs C, Bridel C, Killestein J. 2015. Body fluid biomarkers for multiple sclerosis-The long road to clinical application. Nat Rev Neurol 11: 585596.

Tintore M, Rovira A, Rio J, Tur C, Pelayo R, Nos C, Tellez N, Perkal H, Comabella M, Sastre-Garriga J, et al. 2008. Do oligoclonal bands add information to MRI in first attacks of multiple sclerosis? Neurology 70: 1079-1083.

Tintore M, Rovira A, Arrambide G, Mitjana R, Rio J, Auger C, Nos C, Edo MC, Castillo J, Horga A, et al. 2010. Brainstem lesions in clinically isolated syndromes. Neurology 75: 1933-1938.

Tullman MJ. 2013. Overview of the epidemiology, diagnosis, and disease progression associated with multiple sclerosis. Am J Manag Care 19: S15-S20.

Vollmer T. 2007. The natural history of relapses in multiple sclerosis. J Neurol Sci 256: S5-S13.

Weiner HL. 2008. A shift from adaptive to innate immunity: A potential mechanism of disease progression in multiple sclerosis. J Neurol 255: 3-11.

Weinshenker BG, Bass B, Rice GP, Noseworthy J, Carriere W, Baskerville J, Ebers GC. 1989. The natural history of multiple sclerosis: A geographically based study. 2: Predictive value of the early clinical course. Brain 112: 1419-1428.

Young J, Quinn S, Hurrell M, Taylor B. 2009. Clinically isolated acute transverse myelitis: Prognostic features and incidence. Mult Scler 15: 1295-1302. 


\section{$\&_{\mathrm{CSH}}^{\infty} \&$ Cold Spring Harbor

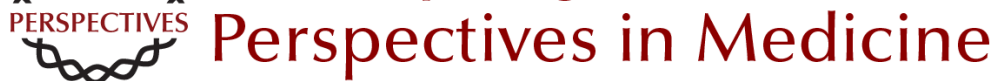

\section{Clinical Course of Multiple Sclerosis}

Sylvia Klineova and Fred D. Lublin

Cold Spring Harb Perspect Med 2018; doi: 10.1101/cshperspect.a028928 originally published online January 22, 2018

\section{Subject Collection Multiple Sclerosis}

Multiple Sclerosis Pathology

Hans Lassmann

Regulatory T Cells: From Discovery to

Autoimmunity

Alexandra Kitz, Emily Singer and David Hafler

The Multiple Roles of B Cells in Multiple Sclerosis

and Their Implications in Multiple Sclerosis

Therapies

Rui Li and Amit Bar-Or

Autologous Hematopoietic Stem Cell

Transplantation in the Treatment of Multiple

Sclerosis

Carolina A. Rush, Harold L. Atkins and Mark S.

Freedman

B-Cell Therapies in Multiple Sclerosis

Joseph J. Sabatino, Jr., Scott S. Zamvil and Stephen L. Hauser

Oral Therapies for Multiple Sclerosis Simon Faissner and Ralf Gold

Interferon $\beta$ for Multiple Sclerosis

Dejan Jakimovski, Channa Kolb, Murali

Ramanathan, et al.

Alemtuzumab as Treatment for Multiple Sclerosis Serafeim Katsavos and Alasdair Coles

\author{
Natalizumab: Perspectives from the Bench to \\ Bedside \\ Afsaneh Shirani and Olaf Stüve \\ Daclizumab Therapy for Multiple Sclerosis \\ Bibiana Bielekova
}

\section{Lifestyle and Environmental Factors in Multiple \\ Sclerosis \\ Lars Alfredsson and Tomas Olsson \\ Biomarkers in Multiple Sclerosis \\ Anu Paul, Manuel Comabella and Roopali Gandhi}
The Evolving Mechanisms of Action of Glatiramer
Acetate

Thomas Prod'homme and Scott S. Zamvil

Regulation of Astrocyte Functions in Multiple Sclerosis

Michael A. Wheeler and Francisco J. Quintana

Experimental Autoimmune Encephalomyelitis

(EAE) as Animal Models of Multiple Sclerosis (MS)

Simon Glatigny and Estelle Bettelli

Neurodegeneration in Progressive Multiple

Sclerosis

Graham Campbell and Don Mahad

For additional articles in this collection, see http://perspectivesinmedicine.cshlp.org/cgi/collection/ 